(2) Open Access Full Text Article

\title{
Human epidermal growth factor receptor
} 2-positive breast cancer: which cytotoxic agent best complements trastuzumab's efficacy in vitro?

This article was published in the following Dove Press journal:

OncoTargets and Therapy

15 June 2013

Number of times this article has been viewed

\section{Tracey Hurrell}

Kim Outhoff

Department of Pharmacology, University of Pretoria, Pretoria, South Africa
Correspondence: Tracey Hurrell Department of Pharmacology, Faculty of Health Sciences, School of Medicine, University of Pretoria, Private Bag X323,

Pretoria 0007, South Africa

$\mathrm{Tel}+27123192663$

Fax +27 I2 319 24II

Email tracey.hurrell@up.ac.za
Introduction: Despite trastuzumab having enhanced selectivity for human epidermal growth factor receptor 2 (HER-2) overexpressing breast cancer cells, treatment is hampered by interindividual variation and tumors with high mitogenic potential. The lack of significant clinical benefit in certain patient cohorts suggests that HER-2 expression is ineffective as a sole prognostic indicator of response to therapy. Therefore, optimizing the clinical role of trastuzumab in drug combinations remains critical for clinical success.

Aim: To investigate the effects of trastuzumab in combination with either doxorubicin or geldanamycin on in vitro cell viability, cell cycling, apoptosis and relative HER-2 expression in HER-2-positive (SK-BR-3) and estrogen receptor-positive (MCF-7) breast adenocarcinoma models.

Results: HER-2-rich SK-BR-3 cells demonstrated a greater sensitivity to the effects of doxorubicin than MCF-7 cells. Concurrent trastuzumab exposure resulted in a further reduction in cell viability. This decreased cell viability induced by doxorubicin was associated with activation of executioner caspases as well as with alterations in cell-cycle kinetics, primarily promoting S-phase accumulation. Doxorubicin had no effect on surface HER-2 density expression. Geldanamycin reduced cell viability significantly greater in SK-BR-3 than MCF-7 cells, and was associated with $\mathrm{G}_{2}$ cell-cycle accumulation. The addition of trastuzumab did not augment these effects. Geldanamycin promoted substantial reductions in relative surface HER-2 density in SK-BR-3 cells.

Conclusion: The in vitro data supported the rationale for using doxorubicin in trastuzumabbased therapies. Therefore, despite the incidence of cardiotoxicity, doxorubicin could retain a fundamental role in treating HER-2-positive breast cancer. While geldanamycin is a potent cytotoxic agent, its concurrent use with trastuzumab requires further research into the transient or permanent nature of alterations in HER-2 status in cell progeny.

Keywords: HER-2, trastuzumab, geldanamycin, doxorubicin, SK-BR-3 cells

\section{Introduction}

Selective amplification of human epidermal growth factor receptor 2 (HER-2) is found in approximately $25 \%-30 \%$ of all primary breast tumor tissue. ${ }^{1}$ HER-2, even when expressed at standard physiological levels, requires no ligand-binding for activation, ${ }^{2-4}$ and is thus the preferred dimer partner for other HER-family members (HER-1, HER3 , and HER-4) due to its constitutive activation. ${ }^{5,6}$ HER-2 plays an essential part in regulating cell proliferation, adhesion, motility, and survival. Therefore, when HER-2 is overexpressed, these cellular responses are exaggerated and unpredictable, ${ }^{4,7,8}$ resulting in a unique, highly mitogenic subtype of breast cancer. ${ }^{1}$ 
The emergence of targeted therapies directed against definitive molecular targets implicated in specific processes of neoplastic transformation has radically altered the natural progression of certain types of cancer. ${ }^{9-11}$ Trastuzumab (Herceptin ${ }^{\circledR}$; Genentech, San Francisco, CA, USA), an antiHER-2 monoclonal antibody, is one such therapy that has had a dramatic impact on the treatment of HER-2-positive breast cancer. ${ }^{9,10}$ However, despite having enhanced selectivity for tissues that overexpress HER-2, ${ }^{12}$ use of this targeted therapy remains hampered by interindividual variation and mitogenic thresholds beyond the limitations of trastuzumab efficacy. ${ }^{11,13}$ Furthermore, although initial studies have suggested that HER-2 status remains stable over time, discordances of up to $30 \%$ are now being reported between primary and metastatic tumor sites. ${ }^{13}$

Retrospective studies suggest that only $30 \%-40 \%$ of patients derive substantial clinical benefit from trastuzumab, ${ }^{11}$ with a diverse set of molecular mechanisms contributing to resistance. ${ }^{14-16}$ Regulatory anomalies resulting in resistance could possibly produce differences in both biological and clinical behavior, further confounding this already heterogeneous disease. ${ }^{16,17}$ This suggests that either HER-2 expression is inadequate as a sole prognostic indicator, or that the fundamental molecular characteristics of the HER-2-positive tumor subtype may rapidly evolve in response to certain stimuli.

Optimizing trastuzumab treatment has been attempted by using sequential combinations of diverse chemotherapeutic agents, ${ }^{16}$ and remains critical to elevate trastuzumab efficacy towards greater clinical success. Herewith, the authors assessed the current relevance and future potential of two cytotoxic agents to determine which model and mechanism of cytotoxicity best complements trastuzumab's efficacy in vitro.

Doxorubicin, an anthracycline currently used in treatment regimens with trastuzumab, is considered one of the most effective antineoplastic agents used to treat breast cancer. ${ }^{9}$ DNA-topoisomerase II- $\alpha$ complex interactions are considered the primary triggering event for both growth arrest and apoptotic event signaling. ${ }^{18,19}$ Although trends towards greater doxorubicin sensitivity have been seen in HER-2 overexpressing cells, it has generally been deemed statistically insignificant. ${ }^{19}$ Furthermore, an undesirable incidence of cumulative dose-dependent cardiotoxicity has become a limiting side effect of this treatment approach..$^{20,21}$

Geldanamycin, a benzoquinone ansamycin, is capable of disrupting the chaperone heat-shock protein 90 (HSP90). ${ }^{22}$ Chaperone proteins are merely mediators in the assembly of polypeptides, and are not ultimately integrated into the assembled structure. ${ }^{23,24}$ Ubiquitous HSP90 protein is constitutively expressed at higher levels in tumor cells compared to normal cell counterparts, suggesting that HSP90 may be a potential target in neoplastic tissue. ${ }^{25}$

HSP90 association is required for the functioning of, amongst others, tumor-suppressor protein $\mathrm{p} 53$, transmembrane tyrosine kinases including the HER family, casein kinase II, and Cdk4/cyclin D complexes. ${ }^{24}$ The degree to which HSP90 is involved in polypeptide assembly correlates with the extent to which the protein and downstream clients are adversely influenced by geldanamycin, and it may therefore only have minimal influence on HER-2.

The aim of this study was to determine which of these two cytotoxic agents best complements the efficacy of trastuzumab in vitro, in order to support the use of doxorubicin with concurrent trastuzumab in HER-2-positive breast adenocarcinoma, and to determine the extent to which geldanamycin reduces downstream HER-2 targets, thereby potentially nullifying its use in trastuzumab-containing regimens.

\section{Materials and methods}

This project was approved by the Faculty of Health Science Student Research Ethics Committee of the University of Pretoria.

\section{Cells and experimental agents}

Adherent breast adenocarcinoma cell lines MCF-7 (American Type Culture Collection HTB-20) expressing normal levels of HER-2 and SK-BR-3 cells (American Type Culture Collection HTB-30) overexpressing HER-2 were maintained in Dulbecco's Modified Eagle's Medium and Roswell Park Memorial Institute 1640 medium (Sigma-Aldrich, St Louis, MO, USA), respectively. Medium was supplemented with $10 \%$ heat-inactivated fetal bovine serum (PAA Laboratories, Pasching, Austria) and 1\% (v/v) penicillin-streptomycin (BioWhittaker; Lonza, Basel, Switzerland). For experimental purposes, cells were seeded at $1 \times 10^{4}$ cells per well or at $1.5 \times 10^{5}$ cells per $25 \mathrm{~cm}^{2}$ flask and maintained in a humidified atmosphere containing $5 \% \mathrm{CO}_{2}$ at $37^{\circ} \mathrm{C}$.

Cell culture-compatible doxorubicin (Sigma-Aldrich) and geldanamycin (Tocris Bioscience, Bristol, UK) were dissolved in dimethyl sulfoxide (Merck, Darmstadt, Germany), stored at $-80^{\circ} \mathrm{C}$, and reconstituted in the appropriate medium prior to use.

Trastuzumab (Herceptin), reconstituted with bacteriostatic water as per the manufacturer's instructions, was kindly donated by Roche Pharmaceuticals 
(Johannesburg, South Africa). Final drug concentrations were as follows: $0.17 \mu \mathrm{M}$ doxorubicin, $0.35 \mu \mathrm{M}$ geldanamycin, and $25-100 \mu \mathrm{g} / \mathrm{mL}$ trastuzumab.

\section{Tetrazolium conversion assay}

After exposure to experimental agents for 96 hours, cell viability was determined using a quantitative colorimetric tetrazolium conversion assay using a 96-well plate format. Vehicle-treated controls, cell-free medium controls, and cellfree drug controls were included to ensure culture sterility and to discern potential spontaneous reactivity of 3-(4,5-dim ethylthiazol-2-yl)-2,5-diphenyl tetrazolium bromide (MTT). Cells were incubated with $20 \mu \mathrm{L}$ MTT solution $(5 \mathrm{mg} / \mathrm{mL}$; Sigma-Aldrich) for 4 hours, washed, dried, and the formazan product solubilized in dimethyl sulfoxide (Merck). Plates were read spectrophotometrically on an ELx800uv universal microplate reader (BioTek Instruments, Winooski, VT, USA) at a dual wavelength of 570 and $630 \mathrm{~nm}$.

\section{Cell-cycle analysis}

Analysis of cell-cycle kinetics was conducted after 24, 48, and 72 hours using intercalating fluorescent propidium iodide with flow-cytometric detection by means of a Cytomics FC500 (Beckman Coulter, Brea, CA, USA). Medium decanted from flasks along with all the cells was fixed in $70 \%$ ethanol and stored overnight at $4^{\circ} \mathrm{C}$. Prior to analysis, the cell pellet was resuspended in $1 \mathrm{~mL}$ of staining solution (propidium iodide [40 $\mu \mathrm{g} / \mathrm{mL}]$, Triton X-100 [0.1\% v/v], DNase-free RNase [100 $\mu \mathrm{g} / \mathrm{mL}$ ]; [Sigma-Aldrich]) and incubated at $37^{\circ} \mathrm{C}$ for 40 minutes. Analysis of histograms was conducted using MultiCycle version 3.0 for Windows deconvolution software (Phoenix Flow Systems, San Diego, CA, USA).

\section{Caspase 3 and 7 assay}

Activated executioner caspases 3 and 7 hydrolyze Ac-DEVDAMC substrates, releasing a fluorescent product (AMC). Cells were exposed for a series of time points between 4 and 30 hours, in a 96-well plate format, followed by removal of medium and addition of $25 \mu \mathrm{L}$ lysis buffer (10 mM HEPES, $1 \mathrm{mM}$ PMSF, 5mM CHAPS [Merck Chemicals, Darmstadt, Germany], $2 \mathrm{mM}$ EDTA and $5 \mathrm{mM} \beta$-mercaptoethanol [Labchem; Johannesburg, South Africa]).

After 40 minutes of incubation on ice, $125 \mu \mathrm{L}$ assay buffer (20 mM HEPES, $2 \mathrm{mM}$ EDTA, and $5 \mathrm{mM} \beta$-mercaptoethanol) containing $5 \mu \mathrm{M}$ ofAc-DEVD-AMC substrate(Sigma-Aldrich) was added to each well. Plates were incubated overnight at $37^{\circ} \mathrm{C}$ to facilitate complete cleavage of Ac-DEVD-AMC substrates and read using a Fluostar Optima (BMG Labtech, Ortenberg, Germany) with excitation-emission wavelengths of 350 and $450 \mathrm{~nm}$, respectively. The fluorescence intensity was standardized to that of the untreated control.

\section{Apoptosis/necrosis}

Later apoptotic hallmarks were assessed using fluorescein isothiocyanate (FITC)-conjugated annexin V after exposure for 48 or 72 hours. Cells were washed and resuspended in annexin $\mathrm{V}$ binding buffer (10 mM HEPES, $150 \mathrm{mM} \mathrm{NaCl}$, $5 \mathrm{mM} \mathrm{KCL}, 1.8 \mathrm{mM} \mathrm{CaCl}_{2}$, and $1 \mathrm{mM} \mathrm{MgCl}_{2}$; Merck) followed by staining with $2.5 \mu \mathrm{L}$ annexin V FITC (SigmaAldrich). Samples were incubated in the dark for 10 minutes. Prior to analysis, $3 \mu \mathrm{L}$ propidium iodide $(3 \mathrm{mM})$ was added as a counterstain for membrane integrity, and data were acquired using the Cytomics FC500 (Beckman Coulter).

\section{Relative HER-2 density}

Relative HER-2 density was determined at 12, 24, and 48 hours using an FITC-conjugated anti-HER-2 affibody molecule (Abcam, Cambridge, UK). Cells were labeled with the affibody molecule to a final concentration of $3.7 \mu \mathrm{g} / \mathrm{mL}$ and incubated on ice for 30 minutes. Samples were washed once before conducting flow-cytometry protocols using the Cytomics FC500 (Beckman Coulter), and the mean relative fluorescence intensity was compared to untreated samples.

\section{Statistics}

A minimum of three independent interday repeats were conducted, with a minimum of three intraday repeats where required. The Kruskal-Wallis nonparametric test was conducted to compare the mean of trastuzumab versus doxorubicin or geldanamycin alone versus the respective combinations. Dunn's multiple-comparison test was used for post hoc analysis, with significance set at $P<0.05$. Statistical analysis was conducted using GraphPad Prism version 5.0 for Windows (GraphPad Software, San Diego, CA, USA).

\section{Results and discussion}

The relationship between HER-2 status and the success of anthracycline-based regimens in patients was initially assessed in the Cancer and Leukemia Group B 8541 study in 1994. ${ }^{26}$ Compilation of retrospective data suggested that HER-2-positive tumors were associated with high overall response rates to high-dose anthracyclines. ${ }^{27}$

Campiglio et al established that SK-BR-3 cells, which also amplify topoisomerase II- $\alpha$, possessed greater sensitivity to the effects of doxorubicin than MCF-7 cells. However, a 
direct correlation between topoisomerase II- $\alpha$ amplification and doxorubicin sensitivity is lacking. Furthermore, doxorubicin sensitivity has been strongly associated with the rate of cellular replication. ${ }^{19}$ Doxorubicin $(0.17 \mu \mathrm{M})$ exposure dramatically decreased cell viability in MCF-7 cells and even more dramatically in HER-2-rich SK-BR-3 cells, which is consistent with the data by Campiglio et al. ${ }^{19}$ Concurrent trastuzumab resulted in a further reduction in cell viability.

This suggests that trastuzumab and doxorubicin possess a relationship that allows each agent to produce an effect without mechanistic interferences by the other (Figure 1). Doxorubicin plays a role in apoptotic cell death during DNA synthesis primarily by influencing the ability of topoisomerase II- $\alpha$ to adequately cut and reanneal DNA. ${ }^{19,28}$ It is known to induce activation of executioner caspases 3 and $7 .{ }^{29}$ These were detected in this study between 12 and 30 hours in both SK-BR-3 and MCF-7 cells (data not shown). Concurrent trastuzumab did not significantly alter the presence or onset of executioner-caspase activity.

Doxorubicin-induced apoptosis was confirmed in both cell lines by detecting the translocation of phosphatidylserine using annexin V binding. Concurrent trastuzumab resulted in a similar distribution of living and apoptotic cells compared to doxorubicin alone. The presence of trastuzumab did not potentiate additional increases in cell death (Figure 4). Thus, the differences in cell viability observed between doxorubicin and the combination may have been due to the antiproliferative effects of trastuzumab complementing the cytotoxic nature of doxorubicin.

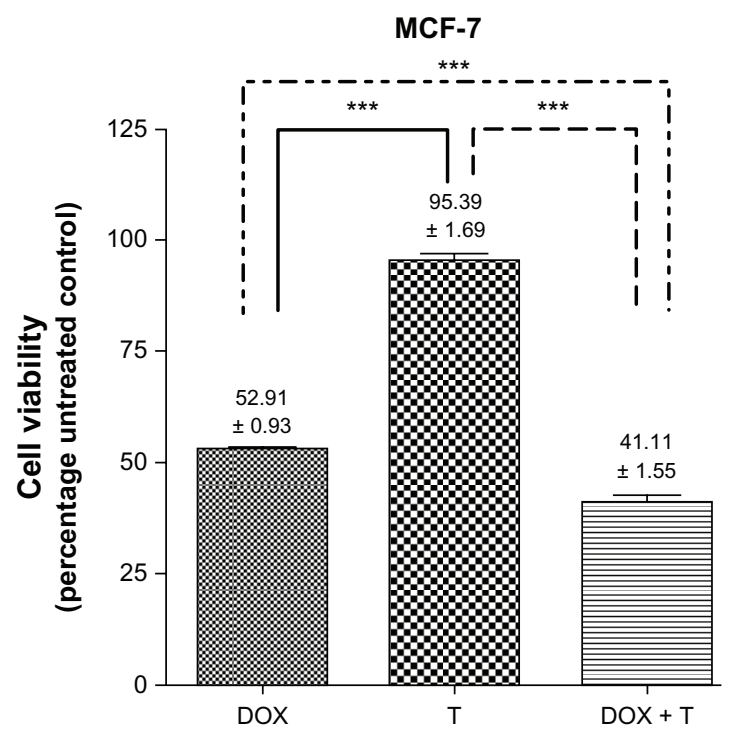

Late S-phase accumulation occurred in MCF-7 cells exposed to doxorubicin alone. While exposure to the doxorubicin-trastuzumab combination maintained a high S-phase accumulation, it also yielded a statistically significant difference in the $\mathrm{G}_{1}$ phase and an insignificant increase in the sub- $\mathrm{G}_{1}$ phase. These alterations in cell-cycle kinetics support the concept of an antiproliferative effect induced by trastuzumab. Similar trends were observed in SK-BR-3 cells (Figure 3). It is known that doxorubicin has the potential to differentially alter cell-cycle kinetics. Cell type-specific interactions result in $\mathrm{G}_{1} / \mathrm{S}$ and $\mathrm{G}_{2} / \mathrm{M}$ checkpoint arrest in some cell types and only $\mathrm{G}_{2} / \mathrm{M}$ checkpoint arrest in others. ${ }^{30}$ Cells in the $\mathrm{G}_{2} / \mathrm{M}$ phase are thought to be the most susceptible to doxorubicin, ${ }^{31}$ which could explain the increase in the percentage of cells in the $\mathrm{S}$ phase in both cell lines.

As a single agent, doxorubicin had no influence on surface HER-2 density. Furthermore, trastuzumab resulted in an equivalent decrease in receptors when used alone and in combination with doxorubicin (Figure 5). Pegram et al demonstrated that HER-2 expression remains unaltered following exposure to doxorubicin, and considered the possibility that cytotoxic drugs such as doxorubicin modify HER-2 functional activity as opposed to altering expression levels. ${ }^{32}$ The consistent decrease in HER-2 by trastuzumab in combination with doxorubicin may have ultimately aided in significantly reducing cell viability. Therefore, the induction of apoptosis by doxorubicin coupled with the cytostatic effects of trastuzumab appeared to provide an effective and complementary treatment modality in vitro.

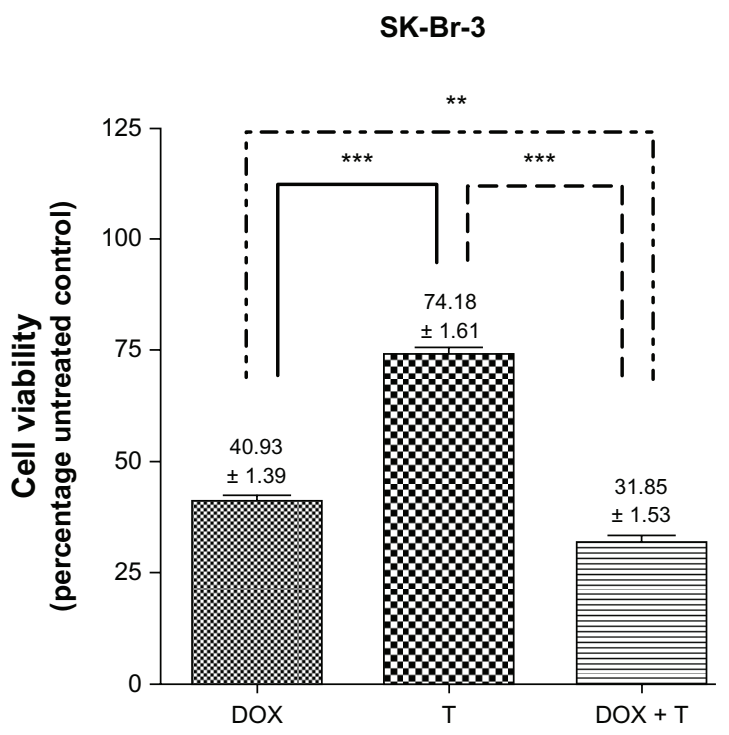

Figure I Cell viability in MCF-7 and SK-BR-3 cells expressed as a percentage of the untreated controls. Statistically significant differences were found between trastuzumab $(\mathrm{T} ; 100 \mu \mathrm{g} / \mathrm{mL}$ ) alone versus doxorubicin (DOX; $0.17 \mu \mathrm{M})$ alone and the DOX-T combination. The combination decreased cell viability more than either agent used alone in both cell lines.

Note: Statistical significance is represented by $* * P<0.01$; *** $P<0.001$. 
Concentration-dependent growth inhibition has also been noted in vivo for doxorubicin-trastuzumab combinations. ${ }^{33}$ Enhancing antitumor activity of targeted therapies remains important, and therefore, despite the limitations of cardiotoxicity, these in vitro results strongly support the use of doxorubicin in trastuzumab-based therapies provided that HER-2 density is maintained throughout disease progression.

Secondly, the authors set out to determine the influence of geldanamycin on HER-2, and its ability to maintain sufficient surface-expression levels to promote an effective combination with trastuzumab. In these experiments, geldanamycin exposure reduced cell viability in MCF-7 cells. Concurrent trastuzumab had negligible effects on these HER-2 normal cells. While cell viability was dramatically lower, the trend for SK-BR-3 cell viability was similar (Figure 2).

Geldanamycin decreases prosurvival factors, which include Akt1, cyclic adenosine monophosphate-dependent protein kinases, and Raf-1, in tumor cell lines. ${ }^{25}$ Raf1 , an integral part of a protein kinase cascade of the mitogen-activated protein kinase signaling pathways, is strongly implicated in reductions in cell proliferation, with cell type-specific recovery from inhibition or depletion being evident. ${ }^{25,34}$ Alternatively, in SK-BR-3 cells, the remarkable decrease in cell viability could have been due to alterations in HER-2 receptors, which are known chaperone clients.

Geldanamycin and a variety of modified analogs, such as herbimycin A and tanespimycin, are suspected inhibitors of normal maturation of tyrosine kinase receptors that include HER-1, insulin-like growth factor receptor, platelet-derived growth factor receptor, and HER-2. ${ }^{35}$ Therefore, the extent of HER-2 depletion on the cell surface was assessed.

Trastuzumab-exposed SK-BR-3 cells illustrated a statistically significant decrease in HER-2 compared to untreated controls. An even greater decrease in HER-2 was observed for geldanamycin alone and the geldanamycin-trastuzumab combination from as early as 12 hours. This trend was sustained at 24 and 48 hours (Figure 6). These results confirmed that HER-2 is a prominent client protein for HSP $90,{ }^{24}$ and that geldanamycin disrupts the ability of HER-2 to accumulate on the cell surface.

Despite the potential complexities, the kinetics of differential molecular responses of a particular cell subtype can be cytostatic or apoptotic. ${ }^{34}$ No executioner caspase 3 or 7 activation was observed in geldanamycin-exposed cells, although apoptosis has previously been observed using the terminal deoxynucleotidyl transferase dUTP nick-end labeling assay, poly(adenosine diphosphate ribose) polymerase cleavage, and proteolytic activation of caspase $3 .{ }^{36}$ Others have observed time-dependent induction of caspase 3 after 48 hours of geldanamycin exposure. ${ }^{37}$ The inability of our assay to accurately quantify executioner-caspase activity may have been an artifact of timing, as our assessments were only carried out until 30 hours post-exposure.

Another possibility was that geldanamycin caused alterations in cell-cycle kinetics prior to executioner caspases being present. ${ }^{38}$ This theory is supported by observations of downregulation of cyclin D1 and cyclin-dependent kinase (CDK)-4 with an accompanying upregulation in CDK inhibitors (p27), prior to detection of apoptosis using
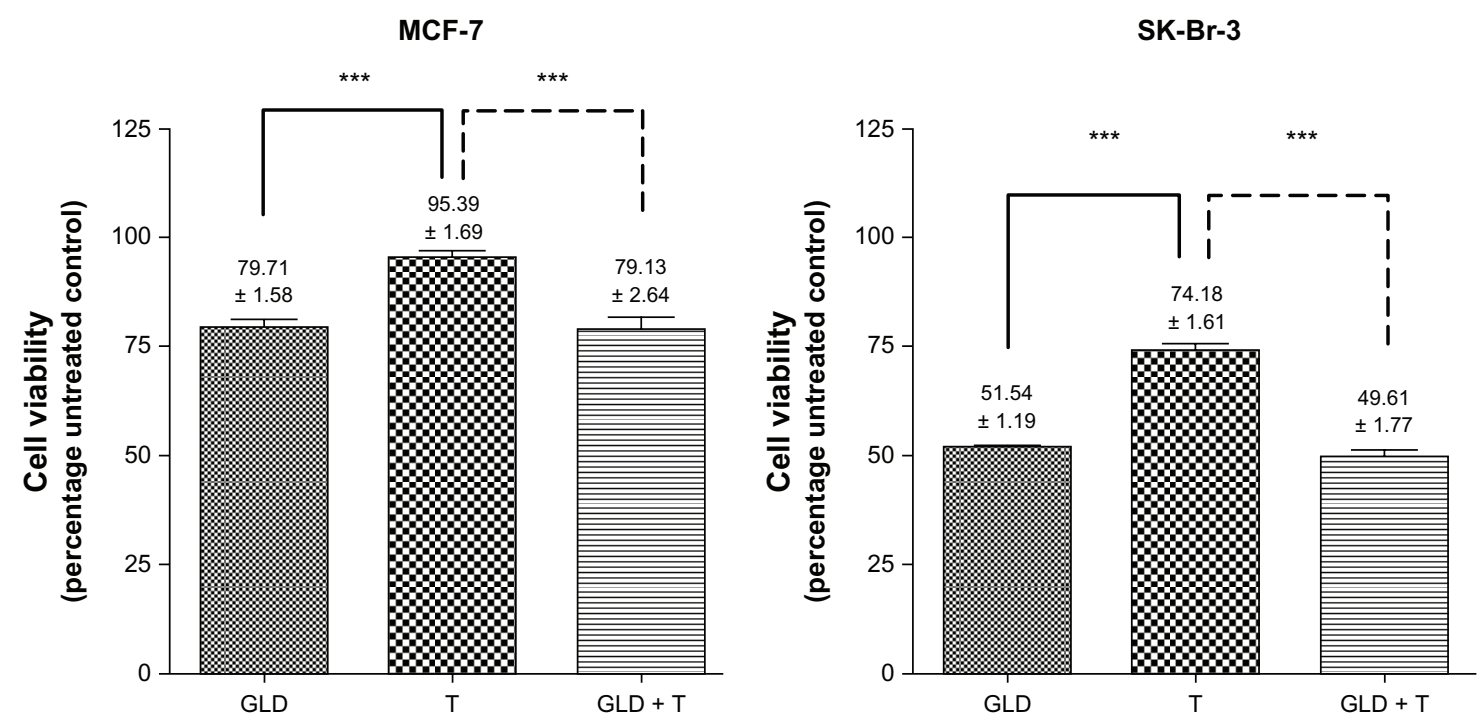

Figure 2 Cell viability in MCF-7 and SK-Br-3 cells expressed as a percentage of the untreated controls. Statistically significant differences were found between trastuzumab ( $\mathrm{T} ; 100 \mu \mathrm{g} / \mathrm{mL}$ ) alone versus geldanamycin (GLD; $0.35 \mu \mathrm{M}$ ) alone and the GLD-T combination.

Note: Statistical significance is represented by $* * * P<0.001$. 

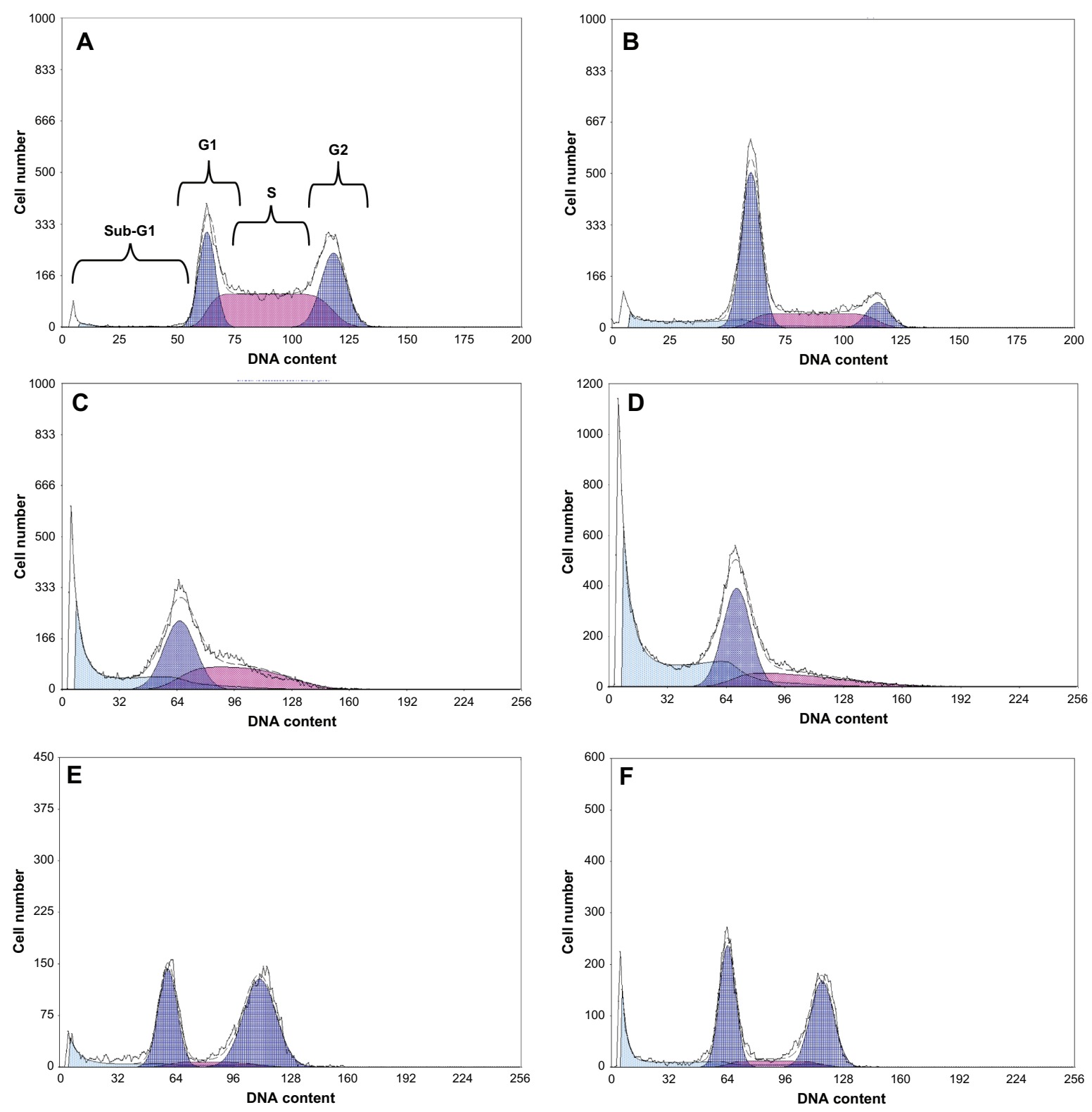

Figure 3 (A-F) Flow-cytometry histograms analyzed with deconvolution software dividing phases into $G_{1}$ (left), S (center), and $G_{2}$ (right). (A) Untreated MCF-7 cells in Dulbecco's Modified Eagle's Medium with 10\% fetal bovine serum; (B) MCF-7 cells exposed to trastuzumab, resulting in G, accumulation at 72 hours; (C) MCF-7 cells exposed to doxorubicin, resulting in late S-phase accumulation at 48 hours; (D) MCF-7 cells exposed to the doxorubicin-trastuzumab combination, resulting in G and late S-phase accumulation at 48 hours; (E) MCF-7 cells exposed to geldanamycin, resulting in $\mathrm{G}_{2}$ accumulation at 48 hours; (F) MCF-7 cells exposed to the geldanamycintrastuzumab combination resulting in $\mathrm{G}_{2}$-phase accumulation at 48 hours. Similar trends in phase accumulation were observed in the SK-BR-3 graphs. However, the percentages differed.

annexin $\mathrm{V}^{39}$ Later hallmarks of apoptosis using annexin V showed increasing apoptosis between 48 and 72 hours in both cell lines. Significant differences were apparent between untreated controls or trastuzumab alone (no effect) versus geldanamycin and the geldanamycin-trastuzumab combination (Figure 4).

Wee1 kinase, a nuclear tyrosine kinase and an integral player in controlling the duration of the sequential $\mathrm{G}_{2}$ cell-cycle phase via CDK2 phosphorylation, is a potential target of HSP90 inhibitors. ${ }^{40}$ Furthermore, antagonizing HSP90 function may result in $\mathrm{G}_{1}$-phase accumulation due to altered CDK4 sequestration and posttranslational destabilization. ${ }^{41}$ Statistically significant $\mathrm{G}_{2}$-phase accumulation was apparent in geldanamycin and geldanamycin-trastuzumab exposed MCF-7 cells from 24 hours. While $G_{2}$ accumulation was the most prominent, increases in the $\mathrm{G}_{1}$ phase were also evident. Similarly in SK-BR-3 cells, geldanamycin promoted comparable perturbations in cell-cycle kinetics. The exception occurred at 72 hours, when differences between geldanamycin alone and the geldanamycin-trastuzumab combination were 

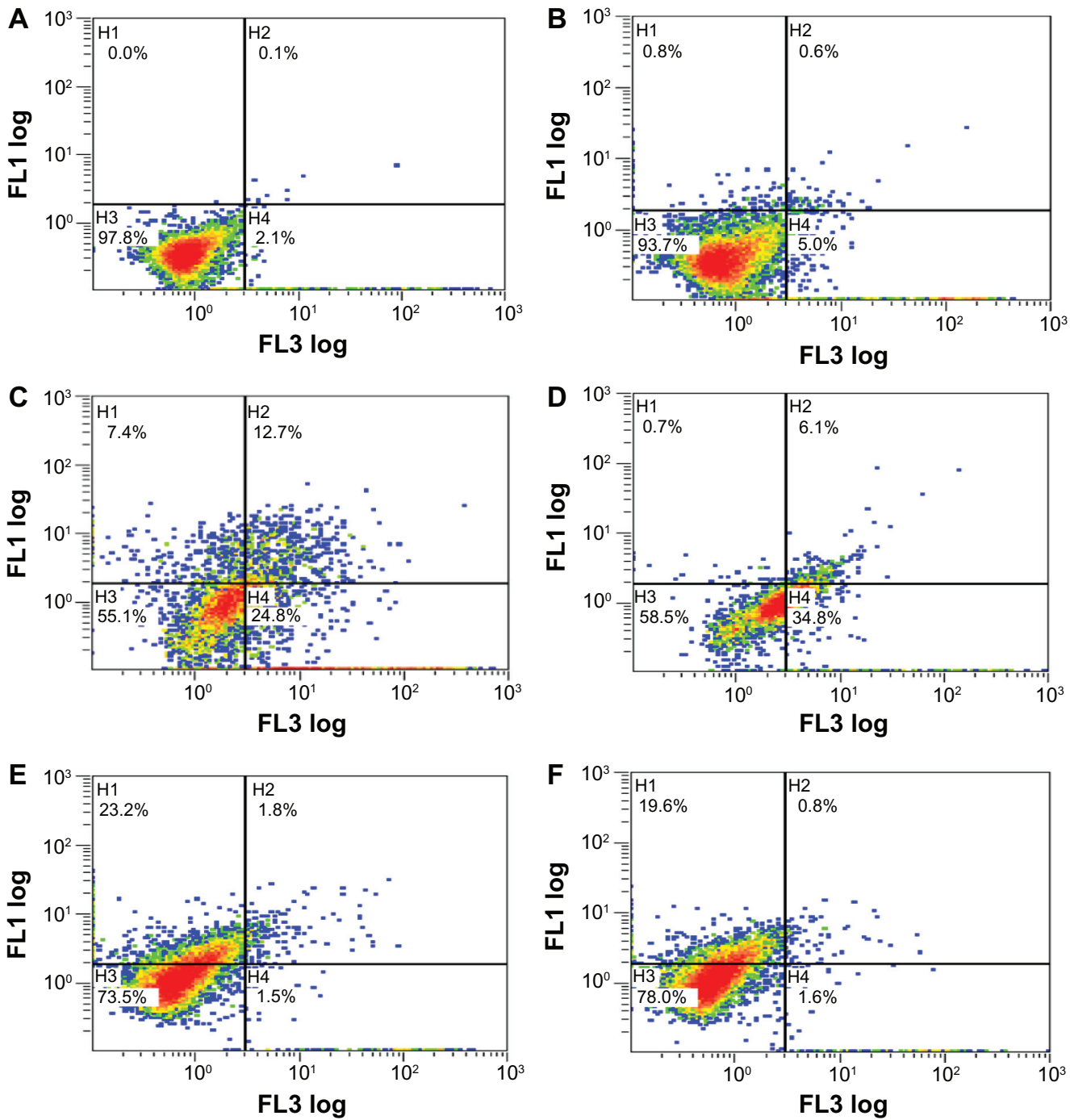

Figure 4 (A-F) Apoptosis/necrosis plots for MCF-7 cells at 72 hours. (A) Untreated control; (B) cells exposed to trastuzumab at 72 hours; (C) cells exposed to doxorubicin at 72 hours; (D) cells exposed to the doxorubicin-trastuzumab combination at 72 hours; (E) cells exposed to geldanamycin at 48 hours; (F) cells exposed to the geldanamycin-trastuzumab combination at 48 hours. Similar trends were observed in the SK-BR-3 graphs. However, higher percentages for necrosis as opposed to early apoptosis were observed.

Notes: FLI log, annexin V fluorescein isothiocyanate; FL3 log, propidium iodide; HI, early apoptosis; H2, late apoptosis; H3, normal cells; H4, necrosis.

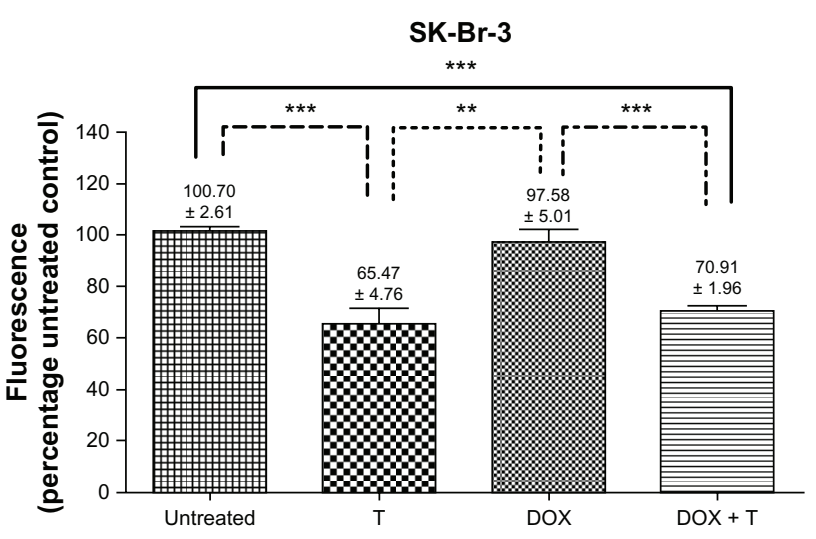

Figure 5 Relative human epidermal growth factor receptor 2 density at 24 hours in SK-BR-3 cells expressed as a percentage of the fluorescence of untreated controls (standardized to 100\%).

Note: Statistical significance followed the same trend at 12 and 48 hours, and is represented by $* * P<0.01$; *** $P<0.001$

Abbreviations: DOX, doxorubicin $(0.17 \mu \mathrm{M})$; T, trastuzumab $(100 \mu \mathrm{g} / \mathrm{mL})$.

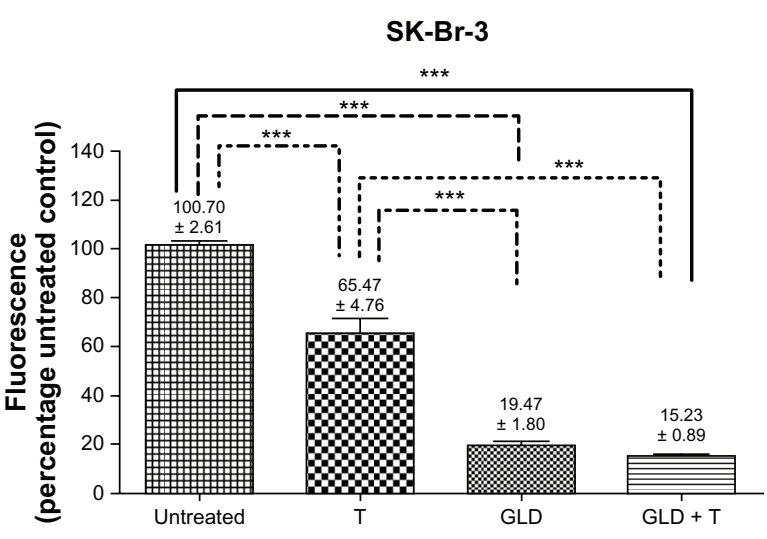

Figure 6 Relative human epidermal growth factor receptor 2 density at 24 hours in SK-BR-3 cells expressed as a percentage of the fluorescence of untreated controls (standardized to 100\%).

Note: Statistical significance followed the same trend at 12 and 48 hours, and is represented by $* * * P<0.001$.

Abbreviations: GLD, geldanamycin $(0.35 \mu \mathrm{M})$; T, trastuzumab $(100 \mu \mathrm{g} / \mathrm{mL})$. 
evident, suggesting a potential influence of trastuzumab on cell-cycle kinetics (Figure 3).

However, the alteration was not translated into differential responses in apoptosis or cell viability, which suggests that the difference was perhaps merely an experimental anomaly due to the deconvolution, or a transient, nontranslatable effect of the combination. Others have shown that geldanamycin-induced decreases in cell viability occur secondary to cell-cycle arrest at both the $\mathrm{G}_{1} / \mathrm{S}$ and $\mathrm{G}_{2} / \mathrm{M}$ boundaries, ${ }^{36}$ which may be unobservable at 72 hours. ${ }^{34}$ Nonetheless, even with two potential points of cell-cycle alterations induced by geldanamycin, there was no potentiation of effects in the presence of trastuzumab. Due to the decrease in HER-2 density, geldanamycin probably functions independently of trastuzumab.

Geldanamycin remains an attractive chemotherapeutic agent, considering its ability to antagonize HSP90 functions crucial for the maintenance of normal housekeeping duties. Differential cell-type responses may be due to different preferential proliferative signaling pathways and the necessity of HSP90 for regulating these functions. The ability of geldanamycin to rapidly and dramatically reduce relative surface HER-2 density indicates that combinatorial regimens with trastuzumab would not have any clinical benefit. However, the ability to regain HSP90 function and for receptor density to be restored upon removal of geldanamycin was not assessed.

\section{Concluding remarks}

The in vitro data support the use of doxorubicin in trastuzumab-based therapies. Despite the enhanced efficacy, the incidence of cardiotoxicity in anthracycline-trastuzumab combinations still casts a shadow over this apparently effective treatment modality. Therefore, if mechanisms for cardioprotection can be identified, this combination could retain its role as an integral component of HER-2-positive breast cancer treatment. Geldanamycin-induced removal of HER-2 as a target for trastuzumab could theoretically result in negligible effects of this targeted therapy in tumor reduction. However, if geldanamycin can be shown to produce only transient alterations in receptor density, and if HER-2 density is regained or maintained in cell progeny, there may still be a place for HSP90 inhibitors as potent cytotoxic agents used subsequently to trastuzumab in treatment regimens.

\section{Acknowledgments}

The authors would like to acknowledge Roche Pharmaceuticals for the kind donation of trastuzumab and the Cancer
Association of South Africa (CANSA) as well as the Research and Development Program (RDP), University of Pretoria, for providing their generous funding, and to Dr AD Cromarty and Dr JJ van Tonder without which this project would not have been possible.

\section{Disclosure}

The authors have no financial disclosures and declare no conflict of interest with regard to this research.

\section{References}

1. Burstein HJ. The distinctive nature of HER2-positive breast cancers. $N$ Engl J Med. 2005;353:1652-1654.

2. Klapper L, Glathe S, Vaisman N, et al. The ErbB-2/HER2 oncoprotein of human carcinomas may function solely as a shared coreceptor for multiple stroma-derived growth factors. Proc Natl Acad Sci U S A. 1999;96:4995-5000.

3. Citri A, Skaria KB, Yarden Y. The deaf and the dumb: the biology of ErbB-2 and ErbB-3. Exp Cell Res. 2003;284:54-65.

4. Ross JS, Slodkowska EA, Symmans WF, Pusztai L, Ravdin PM, Hortobagyi GN. The HER-2 receptor and breast cancer: ten years of targeted anti-HER-2 therapy and personalized medicine. Oncologist. 2009;14:320-368

5. Tzahar E, Waterman H, Chen X, et al. A hierarchical network of interreceptor interactions determines signal transduction by Neu differentiation factor/neuregulin and epidermal growth factor. Mol Cell Biol. 1996;16:5276-5387.

6. Yarden Y, Sliwkowski MX. Untangling the ErbB signalling network. Nature Rev Mol Cell Biol. 2001;2:127-137.

7. Leahy BDJ. Structure and function of the epidermal growth factor (EGF/ ErbB) family of receptors. Adv Protein Chem. 2004;68:1-27.

8. Hudis CA. Trastuzumab - mechanism of action and use in clinical practice. N Engl J Med. 2007;357:39-51.

9. Perez EA, Suman VJ, Davidson NE, et al. Cardiac safety analysis of doxorubicin and cyclophosphamide followed by paclitaxel with or without trastuzumab in the North Central Cancer Treatment Group N9831 adjuvant breast cancer trial. J Clin Oncol. 2008;26:1231-1238.

10. Kruser TJ, Wheeler DL. Mechanisms of resistance to HER family targeting antibodies. Exp Cell Res. 2010;316:1083-1100.

11. Mountzios G, Sanoudou D, Syrigos KN. Clinical pharmacogenetics in oncology: the paradigm of molecular targeted therapies. Curr Pharm Des. 2010;16:2184-2193.

12. Tai W, Mahato R, Cheng K. The role of HER 2 in cancer therapy and targeted drug delivery. J Control Release. 2010;146:264-275.

13. Lower EE, Glass E, Blau R, Harman S. HER-2/neu expression in primary and metastatic breast cancer. Breast Cancer Res Treat. 2009;113:301-306.

14. Nahta R, Yu D, Hung MC, Hortobagyi GN, Esteva FJ. Mechanisms of disease: understanding resistance to HER2-targeted therapy in human breast cancer. Nat Clin Pract Oncol. 2006;3:269-280.

15. Friedländer E, Barok M, Szöllosi J, Vereb G. ErbB-directed immunotherapy: antibodies in current practice and promising new agents. Immunol Lett. 2008;116:126-140.

16. Callahan R, Hurvitz S. Human epidermal growth factor receptor-2positive breast cancer: current management of early, advanced, and recurrent disease. Curr Opin Obstet Gynecol. 2011;23:37-43.

17. Weigelt B, Geyer FC, Reis-Filho JS. Histological types of breast cancer: how special are they? Mol Oncol. 2010;4:192-208.

18. Gewirtz DA. A critical evaluation of the mechanisms of action proposed for the antitumor effects of the anthracycline antibiotics adriamycin and daunorubicin. Biochem Pharmacol. 1999;57:727-741.

19. Campiglio M, Somenzi G, Olgiati C, et al. Role of proliferation in HER2 status predicted response to doxorubicin. Int J Cancer. 2003;105: $568-573$. 
20. Dang C, Fornier M, Sugarman S, et al. The safety of dose-dense doxorubicin and cyclophosphamide followed by paclitaxel with trastuzumab in HER-2/neu overexpressed/amplified breast cancer. J Clin Oncol. 2008;26:1216-1222.

21. Ferenc P, Solár P, Mikeš J, Koval' J, Fedoročko P. Breast cancer and current therapeutic approaches: from radiation to photodynamic therapy. In: Gunduz M, Gunduz E, editors. Breast Cancer: Current and Alternative Therapeutic Modalities. Rijeka, Croatia: InTech; 2011:63-87.

22. Deboer C, Meulman PA, Wnuk RJ, Peterson DH. Geldanamycin, a new antibiotic. J Antibiot. 1970;23:442-447.

23. Toft DO. Recent advances in the study of hsp90 structure and mechanism of action. Trends Endocrinol Metab. 1998;9:238-243.

24. Neckers L, Schulte TW, Mimnaugh E. Geldanamycin as a potential anti-cancer agent: its molecular target and biochemical activity. Invest New Drugs. 1999;17:361-373.

25. Bisht KS, Bradbury CM, Mattson D, et al. Geldanamycin and 17-allylamino-17-demethoxygeldanamycin potentiate the in vitro and in vivo radiation response of cervical tumor cells via the heat shock protein 90-mediated intracellular signaling and cytotoxicity. Cancer Res. 2003;63:8984-8995.

26. Muss HB, Thor AD, Berry DA, et al. c-erbB-2 expression and response to adjuvant therapy in women with node-positive early breast cancer. N Engl J Med. 1994;330:1260-1266.

27. Petit T, Borel C, Ghnassia J, et al. New therapeutic targets in the intrinsic apoptotic pathway in neuroblastoma. Clin Cancer Res. 2001;7: 1577-1581.

28. Wang S, Konorev EA, Kotamraju S, Joseph J, Kalivendi S, Kalyanaraman B. Doxorubicin induces apoptosis in normal and tumor cells via distinctly different mechanisms. J Biol Chem. 2004;279: 25535-25543.

29. Gamen S, Anela A, Pérez-Galán P, et al. Doxorubicin treatment activates a Z-VAD-sensitive caspase, which causes $\Delta \psi_{\mathrm{m}}$ loss, caspase-9 activity, and apoptosis in Jurkat cells. Exp Cell Res. 2000;258:223-235.

30. Bar-On O, Shapira M, Hershko DD. Differential effects of doxorubicin treatment on cell cycle arrest and Skp2 expression in breast cancer cells. Anticancer Drugs. 2007;18:1113-1121.

31. Potter AJ, Gollahon KA, Palanca BJA, et al. Flow cytometric analysis of the cell cycle phase specificity of DNA damage induced by radiation, hydrogen peroxide and doxorubicin. Carcinogenesis. 2002;23: 389-401.
32. Pegram M, Hsu S, Lewis G, et al. Inhibitory effects of combinations of HER-2/neu antibody and chemotherapeutic agents used for treatment of human breast cancers. Oncogene. 1999;18:2241-2251.

33. Baselga J, Norton L, Albanell J, Kim Y, Mendelsohn J. Recombinant humanized anti-HER2 antibody (Herceptin) enhances the antitumor activity of paclitaxel and doxorubicin against HER2/neu overexpressing human breast cancer xenografts. Cancer Res. 1998;58:2825-2831.

34. Hostein I, Robertson D, Distefano F, Workman P, Clarke PA. Inhibition of signal transduction by the Hsp90 inhibitor 17-allylamino-17demethoxygeldanamycin results in cytostasis and apoptosis. Cancer Res. 2001;61:4003-4009.

35. Helmbrecht K, Zeise E, Rensing L. Chaperones in cell cycle regulation and mitogenic signal transduction: a review. Cell Prolif. 2000;33: 341-365.

36. Shimamura T, Lowell AM, Engelman JA, Shapiro GI. Epidermal growth factor receptors harboring kinase domain mutations associate with the heat shock protein 90 chaperone and are destabilized following exposure to geldanamycins. Cancer Res. 2005;65:6401-6408.

37. Kim S, Kang J, Hu W, Evers BM, Chung DH. Geldanamycin decreases Raf-1 and Akt levels and induces apoptosis in neuroblastomas. Int $J$ Cancer. 2003;103:352-359.

38. Park J, Yeh MW, Wong MG, et al. The heat shock protein 90-binding geldanamycin inhibits cancer cell proliferation, down-regulates oncoproteins, and inhibits epidermal growth factor-induced invasion in thyroid cancer cell lines. J Endocrinol Metab. 2003;88:3346-3353.

39. Georgakis GV, Li Y, Younes A. The heat shock protein 90 inhibitor 17-AAG induces cell cycle arrest and apoptosis in mantle cell lymphoma cell lines by depleting cyclin D1, Akt, Bid and activating caspase 9. Br J Haematol. 2006;135:68-71.

40. Aligue R, Akhavan-Niak H, Russell P. A role for Hsp90 in cell cycle control: Wee1 tyrosine kinase activity requires interaction with Hsp90. EMBO J. 1994;13:6099-60106.

41. Stepanova L, Leng X, Parker SB, Harper JW. Mammalian p50 $0^{\text {Cdc37 }}$ is a protein kinase-targeting subunit of Hsp90 that binds and stabilizes Cdk4. Genes Dev. 1996;10:1491-1502.
OncoTargets and Therapy

\section{Publish your work in this journal}

OncoTargets and Therapy is an international, peer-reviewed, open access journal focusing on the pathological basis of all cancers, potential targets for therapy and treatment protocols employed to improve the management of cancer patients. The journal also focuses on the impact of management programs and new therapeutic agents and protocols on

\section{Dovepress}

patient perspectives such as quality of life, adherence and satisfaction The manuscript management system is completely online and includes a very quick and fair peer-review system, which is all easy to use. Visit http://www.dovepress.com/testimonials.php to read real quotes from published authors. 